\title{
Archaeonautica
}

Archaeonautica

L'archéologie maritime et navale de la préhistoire à

l'époque contemporaine

19 | 2017

L'archéologie maritime et navale de la préhistoire à

l'époque contemporaine

\section{Les modèles tridimensionnels de l'épave Dramont $E$. Hydrostatique et réalité virtuelle au service de la restitution en archéologie navale}

Three-dimensional models of the Dramont E shipwreck. Hydrostatics and virtual reality as aids to reconstitutions in naval archaeology

Pierre Poveda

CpenEdition

Journals

Édition électronique

URL : http://journals.openedition.org/archaeonautica/428

DOI : 10.4000/archaeonautica.428

ISSN : 2117-6973

Éditeur

CNRS Éditions

Édition imprimée

Date de publication : 7 décembre 2017

Pagination : $27-40$

ISBN : 978-2-271-11766-3

ISSN : 0154-1854

Référence électronique

Pierre Poveda, «Les modèles tridimensionnels de l'épave Dramont $E$. Hydrostatique et réalité virtuelle au service de la restitution en archéologie navale », Archaeonautica [En ligne], 19 | 2017, mis en ligne le 30 avril 2020, consulté le 30 avril 2020. URL : http://journals.openedition.org/archaeonautica/428 : DOI : https://doi.org/10.4000/archaeonautica.428 


\title{
LES MODÈLES TRIDIMENSIONNELS \\ DE L'ÉPAVE DRAMONT E. \\ HYDROSTATIQUE ET RÉAlitÉ VIRTUELLE AU SERVICE \\ DE LA RESTITUTION EN ARCHÉOLOGIE NAVALE
}

\author{
Pierre POVEDA
}

\begin{abstract}
Résumé
L'apparition dans le champ de l'archéologie de nouveaux outils de recherche permet bien souvent de réinterroger de manière originale des vestiges déjà fouillés et parfois même déjà publiés. L'archéologie navale et plus particulièrement l'épave Dramont $E$ ne font pas exception à cette règle. II s'avère en effet que ce site, pourtant fouillé et étudié de manière remarquable par Claude Santamaria et publié en 1995 dans le $13^{\mathrm{e}}$ numéro d'Archaeonautica, présentait un certain nombre de limites interprétatives que l'on peut désormais pallier en faisant appel aux techniques de restitution informatisées les plus récentes. Ainsi, à travers la production de différents modèles tridimensionnels, des vestiges de la coque restituée ou encore de la cargaison, l'occasion est donnée d'aborder trois problématiques principales qui n'avaient pu être jusqu'alors affrontées que de manière sommaire: forme de la coque, capacité de cargaison et adaptation du navire à sa fonction et à son espace de navigation.
\end{abstract}

Mots-clés: archéologie navale, architecture, épave, restitution 3D, cargaison, hydrostatique

Le recours, de plus en plus fréquent en archéologie navale, à des outils de recherche innovants permet de réinterroger de manière originale des vestiges dont l'étude se trouvait jusqu'alors limitée par le potentiel d'investigation des moyens mis en œuvre lors de leur fouille ou de leur analyse. L'archéologie navale et plus particulièrement l'épave Dramont $E$ ne font pas exception à cette règle ${ }^{1}$. Il s'avère en effet que ce site, pourtant fouillé et étudié de manière remarquable, présentait un certain nombre de lacunes interprétatives auxquelles on pouvait remédier en faisant appel aux techniques de restitution informatisées les plus récentes ${ }^{2}$. Ainsi, à travers la production de différents modèles tridimensionnels, des vestiges, de la coque restituée ou encore de la cargaison, nous avons souhaité interroger trois problématiques principales qui n'avaient

1. Le présent travail est issu d'une thèse de doctorat, réalisée sous la direction de M. Patrice Pomey et soutenue en décembre 2012: Poveda 2012a. Il a aussi fait l'objet d'une publication intermédiaire dans les actes du $12^{\mathrm{e}}$ ISBSA qui s'est tenu à Istanbul en 2009: Poveda 2012b.

2. Nous avons eu recours lors de notre travail à plusieurs logiciels dont les fonctions sont complémentaires: Photoshop $®$, pour la gestion des photographies ainsi que pour les scans de dessins; Illustrator@, pour la vectorisation de ces mêmes dessins et l'illustration 2D plus généralement; Rhinocéros@, pour la C.A.O 3D; Orca3D@, pour les analyses hydrostatiques et de stabilité.

\begin{abstract}
Three-dimensional models of the Dramont $E$ shipwreck. Hydrostatics and virtual reality as aids to reconstitutions in naval archaeology

The appearance of new research tools in the field of archaeology can often lead to the re-examination in a novel manner of vestiges that have already been excavated and sometimes even published. Naval archaeology, and more specifically the Dramont $E$ shipwreck, is susceptible to this new approach. Indeed, despite the fact that this latter site was excavated and studied in a remarkable fashion by Claude Santamaria, and published in 1995 in volume 13 of Archaeonautica, there remained a certain number of gaps in the interpretation, which can now be filled by applying the most recent digital techniques in reconstitution. Thus, through different three-dimensional models of the remains of the reconstituted hull and of the cargo, one can confront three principal issues, which until now have only been treated rather briefly: the form of the hull, the cargo capacity, and the adaptation of the ship to its function as well as to its sailing space.
\end{abstract}

Key words : naval archaeology, architecture, shipwreck, 3D reconstitution, cargo, hydrostatics été jusqu'alors abordées que de manière sommaire, faute de moyens techniques adaptés.

Il s'agissait en premier lieu de tenter d'établir un plan de formes de restitution pour une épave qui en était jusqu'alors dépourvue. Bien que publiée de façon exhaustive par Claude Santamaria en 1995 dans la collection Archaeonautica ${ }^{3}$, une étude de restitution des formes faisait défaut à cette épave qui présentait pourtant bien des arguments favorables à la mise en place d'une telle recherche. D’une part, la quantité de vestiges significatifs était en effet largement suffisante pour produire une restitution des formes dont le degré d'authenticité pouvait s'avérer raisonnable afin de légitimer des analyses postérieures. À cela s'ajoutait que la documentation disponible sur ces vestiges était relativement importante et a priori d'une précision suffisante pour pouvoir lancer un tel processus. D'autre part, au sein du corpus des restitutions d'épaves de la Méditerranée antique, l'épave Dramont $E$ fait office d'exception en raison de sa datation et de ses spécificités architecturales, ce qui

3. Santamaria 1995. Outre cette monographie, relativement exhaustive, on peut mentionner plusieurs articles antérieurs: Tchernia 1969, p. 470-472; Chevalier, Santamaria 1970, p. 356; Joncheray 1975, p. 141-146; Santamaria 1983, p. 57 ; Santamaria 1984, p. 107-114. 
renforçait d'autant la valeur et la nécessité d'une restitution de ces formes.

Dans un second temps, à travers ce travail de restitution, nous avons souhaité tout particulièrement développer la problématique du déplacement ainsi que celle du port en lourd de ce navire afin d'en percevoir l'impact économique potentiel, deux données qui n'avaient fait l'objet que d'estimations globales de la part de $\mathrm{Cl}$. Santamaria. Il était ainsi envisagé de définir bien plus précisément, à travers sa restitution dans le modèle virtuel, l'importance réelle de la cargaison, qui nous était jusqu'ici inconnue à cause des nombreux pillages perpétrés sur ce site.

Enfin, il s'agissait, à travers les résultats obtenus conjointement par l'analyse hydrostatique et de stabilité et par la restitution de la cargaison, d'affiner notre connaissance du navire et d'évaluer son adaptation à l'espace de navigation envisagé.

\section{PRÉSENTATION}

Leépave Dramont E représente incontestablement un jalon remarquable de l'histoire maritime de la Méditerranée occidentale. Chargée notamment de grandes amphores cylindriques africaines du type Keay XXXV, de vaisselle en sigillée africaine D et d'un petit trésor monétaire, ce site se révèle en effet comme un témoin privilégié, par sa conservation et sa nature, de la dynamique développée au début du v ve ap. J.-C. par les Vandales dans l'ancienne province d'Afrique, devenue Royaume Vandale (Santamaria 1995, p. 191-192). Pour ce qui est de son histoire moderne, on retiendra que l'épave a été découverte en 1965 par 42 mètres de fond à l'ouest du Cap Dramont à Saint-Raphaël dans le Var (Tchernia 1969, p. 470-472) et qu'elle fut, à partir de cet instant, abondamment pillée jusqu'au lancement, au début des années quatre-vingt, d'une fouille exhaustive entreprise sous la direction de Claude Santamaria durant onze campagnes (Fig. 1).

La partie conservée de la coque, qui correspond en majorité au côté tribord du navire, s'étend en longueur sur une distance de $10,04 \mathrm{~m}$ et sur $5 \mathrm{~m}$ en largeur (dont 3,18 m uniquement pour le côté tribord). En plus de la charpente axiale, constituée de la quille d'une longueur de 7,09 m et des deux fragments du brion d'étrave et de celui de l'étambot, on note aussi la présence sur le bordé de trente niveaux de virures (douze à bâbord et dix-huit à tribord) et de trente-cinq couples au niveau de la charpente transversale. À cela viennent s'ajouter de nombreux éléments internes tels que l'emplanture, deux carlingots, des cloisons, le puits de la pompe de cale ainsi qu'une importante quantité de vaigres.

Du point de vue de sa structure, ce navire, construit sur «bordé premier $»^{4}$, se caractérise par un bordé simple relié à franc-bord par un jeu de tenons et mortaises, une membrure composite alternant varangues et demi-couples, avec ponctuellement des couples outrepassés, la présence de varangues brochées et enfin l'usage d'un massif d'emplanture posé sur le dos des membrures par le biais de carlingots. Tous ces éléments tendent à rapprocher Dramont $E$ du type architectural dominant de la période impériale romaine, tel qu'il a été caractérisé par Patrice Pomey (Pomey 1998, p. 68-69; Pomey, Rieth 2005, p. 166-167). Cependant, du point de vue morphologique, la section transversale à fort retour de galbord rappelle le type architectural prédominant à la période

4. On soulignera que cette épave est par ailleurs un des exemples les plus tardifs de la construction sur bordé (Pomey et al. 2012, p. 256-257). hellénistique et républicaine. On serait donc en présence d'un nouveau type architectural, combinant la géométrie de forme du type hellénistique à la structure du type impérial, dont l'origine et le contexte culturel restent à définir ${ }^{5}$. Les caractéristiques de ce nouveau type se retrouvent par ailleurs sur l'épave Saint-Gervais 2 qui témoigne, deux siècles plus tard, d'un changement radical du processus de construction (Pomey et al. 2012, p. 264-266, 302303).

Outre la monographie qui lui est consacrée, nous avons pu disposer d'une importante quantité de photographies complémentaires. Les relevés consistent en seize coupes transversales, produites au droit des couples, trois planimétries, une coupe longitudinale, ainsi que de multiples relevés de détails effectués sur différentes parties du navire remontées et étudiées à la surface 6 .

\section{ÉTABLISSEMENT DES DIMENSIONS ET DU PLAN DE FORMES}

\section{RECHERCHE DES FORMES INITIALES}

Le navire a été découvert gisant sur son flanc droit selon une gîte de $16^{\circ}$ environ. Cette disposition a permis d'une part la bonne conservation du flanc tribord, d'autre part une meilleure conservation des formes de la carène dans cette partie, celle-ci pouvant opposer au poids de la cargaison le soutien du fond sableux. À l'inverse, le côté bâbord n'est que très partiellement conservé et fortement déformé, le poids des amphores et son ensablement plus tardif ne permettant pas une conservation optimale. Au niveau des extrémités, l'importance des forces appliquées à la structure a amené une ouverture plus rapide du bordé ainsi qu'une déformation plus marquée de la charpente transversale.

Plus ponctuellement cependant, il est apparu bien difficile de déterminer la présence de déformations et de quantifier leur importance. Sur les coupes transversales on remarque ainsi les nombreuses divergences d'angle par rapport au droit de la quille entre les pattes tribord et bâbord d'un même couple, les plus importantes différences étant localisées sur les couples M18, M19, M21, M27 et M33. Cl. Santamaria évoque à ce propos certaines asymétries dans le façonnage des chanfreins du bord supérieur de la quille pouvant nous amener à conclure à une asymétrie ponctuelle de la carène, hypothèse envisageable dans le cadre d'une construction sur bordé premier. Toutefois, les divergences bâbord/tribord sont ici tellement marquées qu'il apparaît plus prudent de tabler sur une ouverture prononcée du flanc bâbord amenant à l'écrasement de l'ensemble des structures transversales sur ce côté.

Le dressement du plan des formes du navire du cap Dramont s'est donc révélé fort peu aisé et a nécessité, plus que d'ordinaire, une remise en question permanente des résultats, qui s'est traduit par la production de trois versions différentes du plan de formes de restitution. Afin de comparer la validité et la cohérence d'ensemble de ceux-ci, nous avons tenté à partir des différents relevés, planimétrique et en coupe, de recréer à l'aide du logiciel Rhinocéros ${ }^{\mathrm{TM}}$ un modèle virtuel tridimensionnel des vestiges, le but étant de

5. Pomey 2004, p. 370-373; Pomey, Rieth 2005, p. 163-165; Pomey et al. 2012, p. $256-257$

6. Après consultation des carnets de fouille dans les archives du DRASSM, il s'est avéré que les relevés présentés dans la publication sont identiques et en même nombre que ceux se trouvant dans les rapports. 


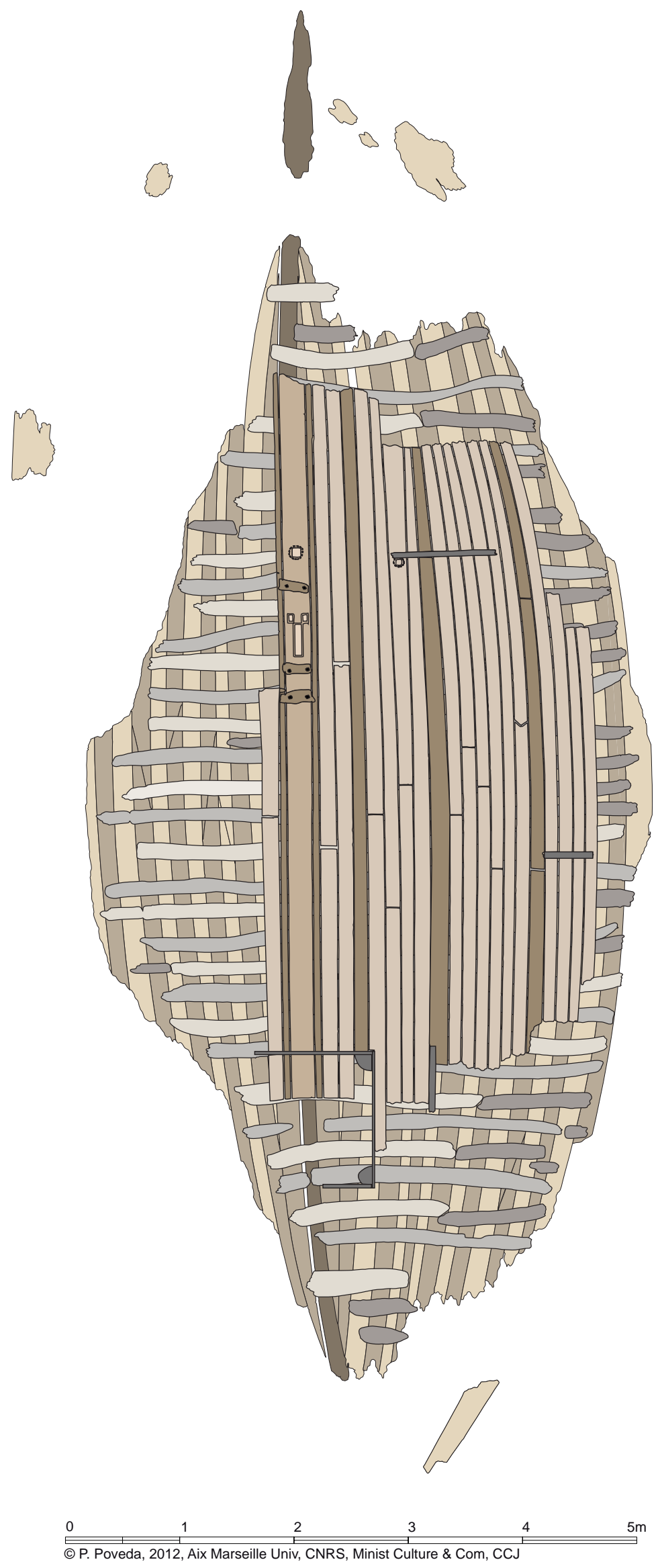

Fig. 1: Planimétrie des vestiges (P. Poveda d'après Santamaria 1995). 
retrouver le basculement de chaque coupe transversale en nous appuyant sur l'harmonisation dans les trois vues de l'ensemble des relevés. Il est vite apparu que seul un basculement hétérogène et spécifique de chacun des couples permettait d'obtenir une cohérence accrue entre les différents relevés et l'obtention d'une forme de carène cohérente à même de se prêter à une tentative de restitution (Fig. 2). C'est sur cette base de redressement transversal ainsi que sur un remontage de la coupe longitudinale que nous nous sommes donc fondés afin de projeter nos élancements en respectant au maximum l'allure des lignes d'eau décrite par les vestiges redressés.

\section{DÉFINITION DES DIMENSIONS PRINCIPALES}

Comme c'est bien souvent le cas, c'est la détermination de la hauteur du livet de pont qui demeure la question la plus difficile à résoudre. Compte tenu de la préservation d'une grande partie du matériel amphorique, la restitution de l'ensemble de la cargaison dans le modèle virtuel se présentait comme l'une des solutions les plus adaptées pour nous indiquer le niveau minimal nécessaire de restitution du livet, afin que la cale présente des dimensions suffisantes pour accueillir l'ensemble de cette cargaison. Cette donnée peut être couplée à la restitution d'un certain nombre de préceintes répétant le schéma observé sur les parties conservées.

\section{Recherche d'une hauteur sous barrot à partir de la restitution de la cargaison}

C'est essentiellement au niveau du maître-couple (M19 - M20) que nous avons recherché la mesure minimale nécessaire pour le placement d'une ou de deux couches d'amphores en prenant comme base de calcul le module le plus grand des amphores Keay XXXV7, d'une hauteur de $107 \mathrm{~cm}$.

Sur l'axe de quille tout d'abord, la restitution d'une unique couche d'amphores nécessite une hauteur sous barrot de $1,10 \mathrm{~m}$ tandis que la restitution d'un double niveau demande une hauteur sous barrot de $1,85 \mathrm{~m}^{8}$. On notera qu'aux extrémités avant et arrière, la répartition des amphores dans la cale devait être plus problématique compte tenu du refermement de la carène: la présence d'un deuxième niveau y est donc complètement à exclure, et ce quelle que soit la hauteur de restitution du pont (cf. infra et Fig. 3). Vers les flancs bâbord et tribord, la hauteur sous barrot devait aussi nécessairement s'établir aux alentours d'une centaine de centimètres afin de permettre d'y accueillir les amphores Keay XXXV retrouvées bien en place en périphérie de la cargaison. Cela a pour conséquence principale, quelle que soit l'hypothèse, d'augmenter d'une trentaine de centimètres la hauteur sous barrot minimale au niveau de l'axe de quille.

Compte tenu de ces différents éléments on peut donc résumer les deux hypothèses de chargement de la façon suivante: la restitution d'un unique niveau d'amphores commande d'avoir une hauteur sous barrot de 1,40 m sur l'axe de quille, permettant de replacer correctement la cargaison retrouvée tant sur l'axe de quille que vers les flancs. Dans cette configuration, le navire présente alors une hauteur entre le bas de quille et le livet d'environ $1,90 \mathrm{~m}$. La restitution d'un second niveau nécessite, quant à elle,

7. Nous prenons ici volontairement en référence l'amphore complète la plus grande, inventoriée comme A. 01/81 (Santamaria 1995, p. 27 et p. 30, Pl. I).

8. Mesuré graphiquement sur l'empilement d'amphores dans le modèle virtuel. une hauteur minimale entre 1,48 et 1,67 m sur l'axe de quille. On notera que la possible présence d'un bouge du pont devrait engendrer une hauteur sous barrot légèrement plus importante vers le centre du navire le long de l'axe de quille ${ }^{9}$, suffisant en tout cas pour pouvoir y intégrer, sur une certaine surface, les deux niveaux d'amphores dont la hauteur totale devait atteindre $1,85 \mathrm{~m}^{10}$. On peut ainsi estimer pour cette seconde hypothèse que la hauteur de livet devait s'établir, depuis le bas de quille, entre $1,98 \mathrm{~m}$ et $2,17 \mathrm{~m}$.

\section{Recherche d'une hauteur sous barrot à partir de la restitution de différents niveaux de préceintes}

Afin d'affiner notre hypothèse de restitution, nous avons tenté de définir une hauteur du livet en nous basant sur la restitution pour la muraille de trois, quatre ou cinq niveaux de préceintes et de virures intermédiaires dont les dimensions seraient comparables à celles encore conservées.

On constate que la restitution de trois niveaux engendrerait la restitution d'une hauteur totale entre le bas de quille et le livet de $1,90 \mathrm{~m}$. Dans ces conditions, on se rapproche très fortement des conditions déduites pour la restitution d'un seul niveau d'amphores. Soulignons cependant que dans cette configuration le navire se trouve déjà plein avec les seules amphores rescapées du pillage (environ 400) et inventoriées en début de fouille en 1981.

Dans le cas de quatre niveaux de préceintes, on restitue une hauteur totale entre le bas de quille et le livet de 2,19 m (Fig. 4). Avec une telle hauteur sous barrot, la cale serait à même de recevoir deux niveaux d'amphores, pouvant correspondre aux amphores recueillies durant la fouille au niveau inférieur, ainsi qu'aux éventuelles amphores pillées pour le niveau supérieur.

Enfin, la restitution de cinq niveaux de préceintes engendrerait une hauteur totale entre le bas de quille et le livet de 2,55 m. Dans ce cas, les dimensions de la cale ne semblent pas s'accorder avec l'importance constatée des vestiges de la cargaison, ce qui nous a amené rapidement à écarter cette dernière hypothèse.

Nous avons donc naturellement privilégié les deux premières hypothèses en estimant que la comparaison entre les données propres aux diverses restitutions du chargement et de la coque avec les résultats de l'étude hydrostatique et de stabilité permettrait d'affiner notre connaissance du navire et de son adaptation à l'espace de navigation envisagé.

En nous appuyant donc sur des hauteurs de livet de $1,90 \mathrm{~m}$ et $2,19 \mathrm{~m}$, correspondant res pectivement à la restitution de trois ou quatre niveaux de préceintes, nous avons placé notre livet de pont au niveau du maître-couple, livet que nous avons par la suite prolongé jusqu'aux massifs d'extrémité. La tonture du pont est quant à elle déterminée par l'allure des cinq dernières virures conservées. En fin de compte, nous obtenons pour les deux hypothèses les dimensions principales suivantes:

9. Comme observé sur le fragment conservé de pont de l'épave 2 de l'anse des Laurons (Gassend et al. 1984, p. 78).

10. Cette hauteur de $1,85 \mathrm{~m}$ peut être obtenue si l'on tient compte de l'existence d'un bouge du pont qui serait établi entre $36,5 \mathrm{~cm}$, équivalent au $1 / 17^{\mathrm{e}}$ de la largeur au maître-couple comme sur l'épave Laurons 2 (Gassend et al. 1984, p. 78), et $17,8 \mathrm{~cm}$, équivalent au $1 / 35^{\mathrm{e}}$ de la largeur au maître-couple, comme sur l'épave Napoli A (Poveda 2012, p. 154). 


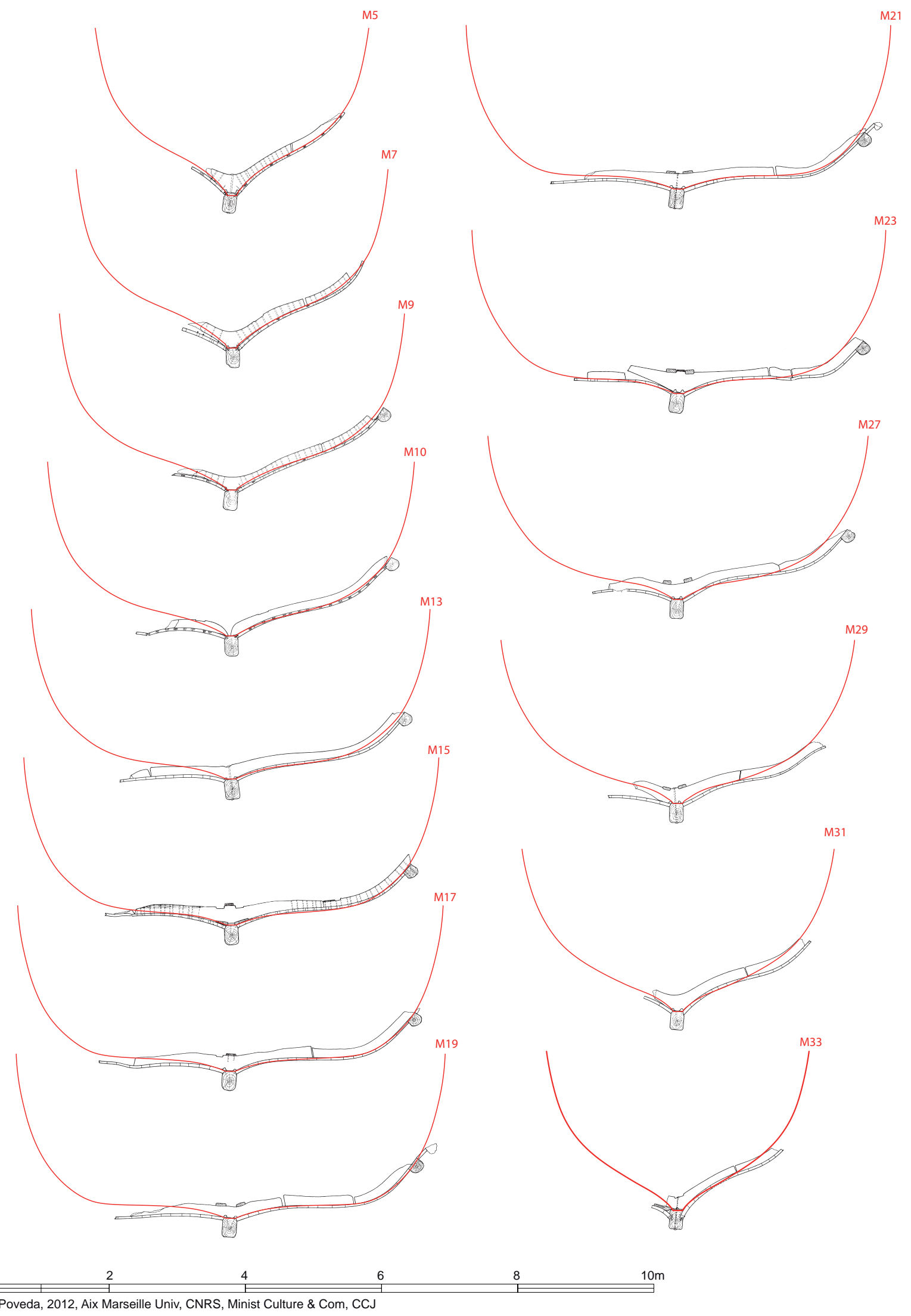

Fig. 2: Vue de la remise en place des coupes transversales des vestiges sur les coupes du plan de formes de restitution (P. Poveda). 


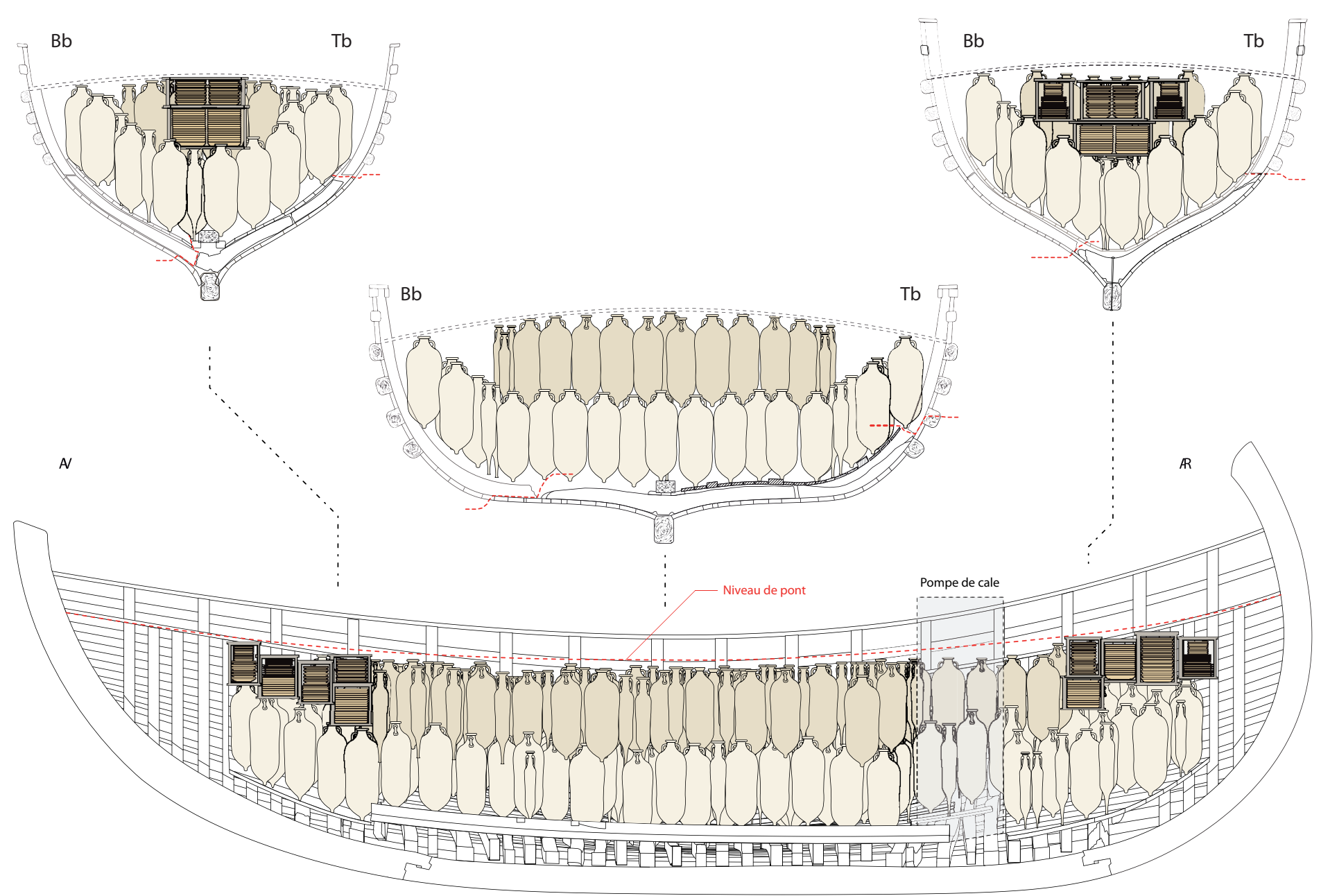

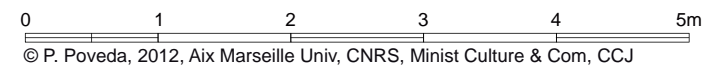

Fig. 3: Schéma de répartition des deux niveaux d'amphores de la cargaison d'amphores et des céramiques sigillées dans la deuxième hypothèse de restitution du navire (P. Poveda).

Tableau récapitulatif des dimensions des deux hypothèses de restitution

\begin{tabular}{|c|c|c|}
\hline & Hypothèse 1 & Hypothèse 2 \\
\hline Longueur HT & $\mathbf{1 4 , 1 0 ~} \mathbf{~}$ & $\mathbf{1 4 , 1 0 ~} \mathbf{~}$ \\
\hline Longueur entre perpendiculaires & $\mathbf{1 3 , 2 6} \mathbf{~}$ & $\mathbf{1 3 , 4 6} \mathbf{~}$ \\
\hline Largeur MC max (préceintes comprises) & $\mathbf{6 , 3 9} \mathbf{~}$ & $\mathbf{6 , 4 4} \mathbf{~}$ \\
\hline Largeur MC (face int.) & $\mathbf{6 , 1 0 ~} \mathbf{~}$ & $\mathbf{6 , 2 1} \mathbf{~}$ \\
\hline Creux au MC (Vaigrage-Livet) & $\mathbf{1 , 4 0 ~} \mathbf{~}$ & $\mathbf{1 , 5 7} \mathbf{~}$ \\
\hline Hauteur bas de quille-pavois (BQP) & $\mathbf{1 , 9 0 ~} \mathbf{m}$ & $\mathbf{2 , 1 9} \mathbf{~}$ \\
\hline Rapport L / & 2,17 & 2,17 \\
\hline Rapport I / & 4,36 & 3,94 \\
\hline Rapport L / h & 9,47 & 8,55 \\
\hline Rapport I / h BQP & 3,21 & 2,83 \\
\hline Rapport L/ h BQP & 6,97 & 6,15 \\
\hline
\end{tabular}

\section{RESTITUTION}

స్
Compte tenu des approximations et des manques qui apparaissent à travers l'analyse et le replacement des différents relevés de terrain sur le modèle tridimensionnel des vestiges, nous avons estimé qu'une restitution très détaillée du navire ne s'imposait pas. Nous nous sommes donc contenté d'un modèle simplifié dans laquelle certaines parties sont définies précisément (charpente axiale, bordé, charpente transversale, vaigrage, charpente interne) tandis que d'autres (pont, gréement) ne font l'objet que d'une simple ébauche (Fig. 6). L'ensemble nous permet d'établir un devis de poids global du navire suffisamment précis pour entreprendre les analyses de stabilité souhaitées. Il n'en va pas de même pour la cargaison qui a fait l'objet d'une restitution beaucoup plus soignée (Fig. 7).

\section{LA CARGAISON (Fig. 3 et 7)}

Nous avons pu voir précédemment que la nature et l'importance de la cargaison ont été des éléments majeurs dans l'établissement des caractéristiques dimensionnelles de nos deux hypothèses de restitution. Nous reviendrons ici plus précisément sur la manière dont nous avons abordé la production, dans le modèle tridimensionnel, d'une restitution qui soit la plus complète et la plus fidèle possible de l'ensemble de la cargaison. Il s'agissait, au-delà de la simple étude des poids et de leur répartition au sein du navire de 


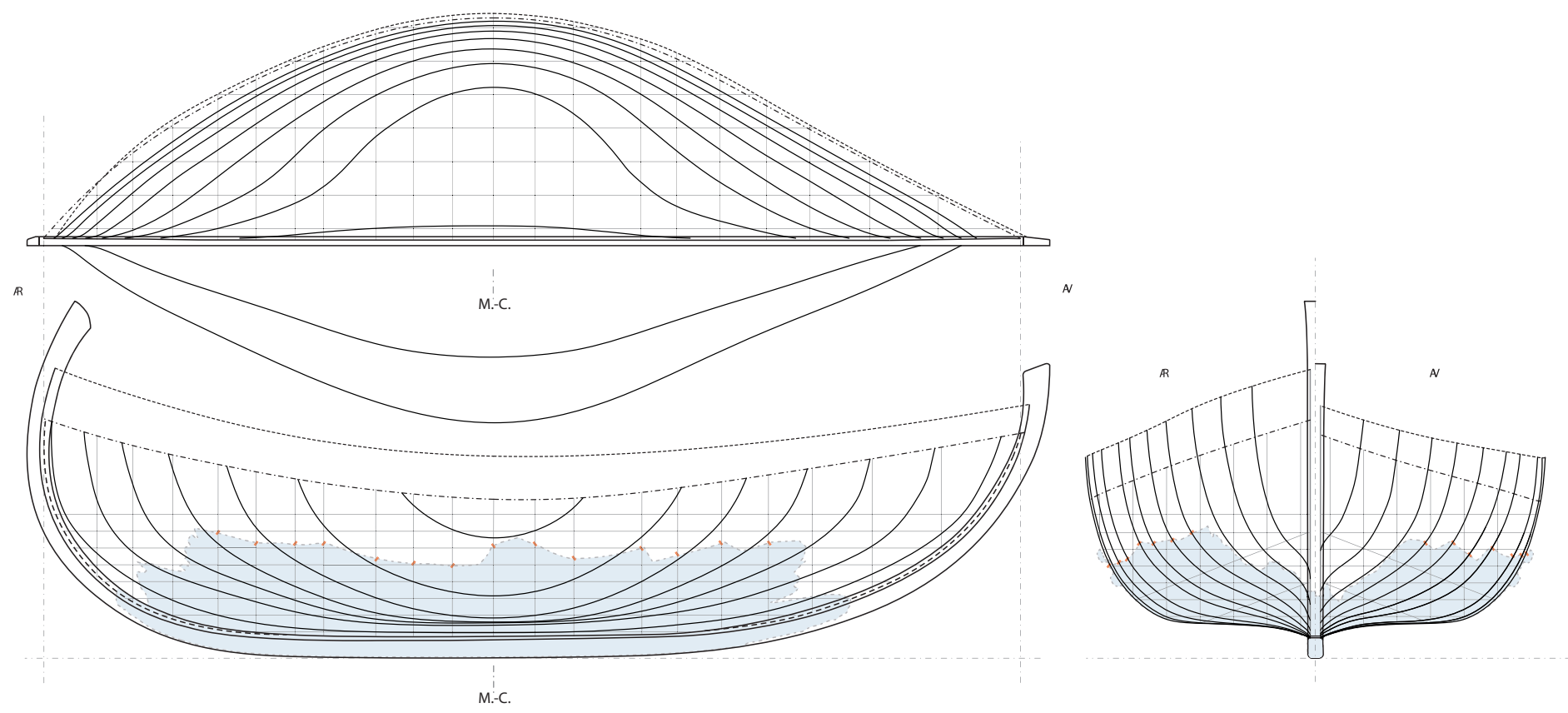

$\begin{array}{lllll}0 & 2 & 4 & 8 & 8\end{array}$

Fig. 4: Plan de formes de la deuxième hypothèse de restitution (P. Poveda).

pouvoir apprécier l'adaptation de la cale ainsi restituée à la quantité et à l'importance du chargement d'amphores et de céramiques constatées durant la fouille.

L'une des problématiques principales de la restitution de la cargaison était de pouvoir vérifier dans quelle mesure la présence d'une seconde couche d'amphores pouvait se justifier à la fois par l'étude de l'espace de cale disponible mais aussi par l'estimation de l'enfoncement et de la stabilité du navire. À l'image des deux hypothèses de restitution pour la carène, nous proposerons donc ici aussi deux hypothèses pour la cargaison: l'une reposant sur un unique niveau d'amphores, pouvant correspondre aussi bien à la restitution de trois ou quatre niveaux de préceintes, et une seconde dans laquelle un second niveau d'amphores vient s'ajouter au premier, situation correspondant uniquement à la restitution de quatre niveaux de préceintes.

\section{Restitution du premier niveau d'amphores}

Le replacement des amphores conservées du premier niveau dans le modèle tridimensionnel de restitution s'est basé sur la planimétrie de la fouille de la cargaison ainsi que sur la description et le relevé des différents modules découverts et dont $\mathrm{Cl}$. Santamaria fait une description minutieuse (Santamaria 1995, fig. 9). Les limites aux extrémités pour la restitution de ce premier niveau du chargement ont été déterminées à partir de l'emprise maximum des amphores Keay XXXV retrouvées en place. Sur l'avant, les amphores $\mathrm{n}^{\circ} 9$ et $\mathrm{n}^{\circ} 2$, tout comme les $\mathrm{n}^{\circ} 121$ et $\mathrm{n}^{\circ} 134$ sur l'arrière, nous ont paru définir des bornes vraisemblables, reprises dans notre modèle de restitution. On constate alors la présence de deux espaces libres vers les extrémités qui peuvent être associés, malgré leur taille réduite, à des lieux de vie et de stockage pour l'équipage.

Malgré la diversité morphologique des groupes d'amphores, nous avons considéré que la restitution de deux modules de Keay XXXV, un moyen (Haut. $97 \mathrm{~cm}$; Diam. $37 \mathrm{~cm}$ ) et un grand (Haut. $100 \mathrm{~cm}$; Diam. $39 \mathrm{~cm}$ ) serait suffisante pour notre démarche. De la même manière pour les amphores cylindriques de petit diamètre, si l'on peut distinguer plusieurs variantes, nous avons fait le choix de nous limiter à deux modules médians représentant d'une part les Keay XXV (Haut. $100 \mathrm{~cm}$; Diam. $18 \mathrm{~cm}$ ) et d'autre part, les spatheia (Haut. $83 \mathrm{~cm}$; Diam. $13 \mathrm{~cm}$ ). Enfin, nous avons émis l'hypothèse que la position des amphores lors du relevé devait représenter une situation proche du chargement originel et que l'on pouvait donc replacer directement à l'intérieur de la cale restituée les amphores dans leur position de découverte. On remarquera, comme $\mathrm{Cl}$. Santamaria, que la répartition générale des amphores dans la cale ne s'effectue pas de manière homogène et que l'on distingue très clairement des schémas de rangement quelquefois en quinconce, quelquefois en carré, qui traduisent une nécessaire adaptation aux divers modules d'amphores à arrimer. On note aussi l'adaptation du chargement aux divers obstacles propres à la cale de Dramont E, parmi lesquels on distingue notamment le puits de la pompe de cale, les épontilles et le fourreau d'étambrai ${ }^{11}$.

La restitution de ce premier étage dans le modèle tridimensionnel s'avère, en fin de compte, tout à fait compatible avec l'espace de cale défini dans les deux hypothèses de restitution et l'on constate même que l'on peut se permettre de combler certaines lacunes, notamment aux extrémités, qui peuvent être mises sur le compte des pillages. On notera toutefois que, dans la première hypothèse de restitution de la carène, pour laquelle nous avons proposé une hauteur sous barrot de 1,40 m, le calage des amphores situées vers les flancs se révèle légèrement problématique. On ne peut en effet y parvenir qu'en plaçant les amphores de plus petit module $(80 \mathrm{~cm}$ de hauteur). Concernant le décompte total d'amphores, on peut dresser un tableau relativement proche de ce qui a pu être découvert lors de la fouille: avec 218 à 222 amphores de type Keay XXXV pour 184 conservées, 120 petits spatheia pour 108 conservés, et

11. Phénomène qui avait déjà été clairement mis en évidence sur la fouille de la Madrague de Giens (Tchernia et al. 1978, p. 19-25). 

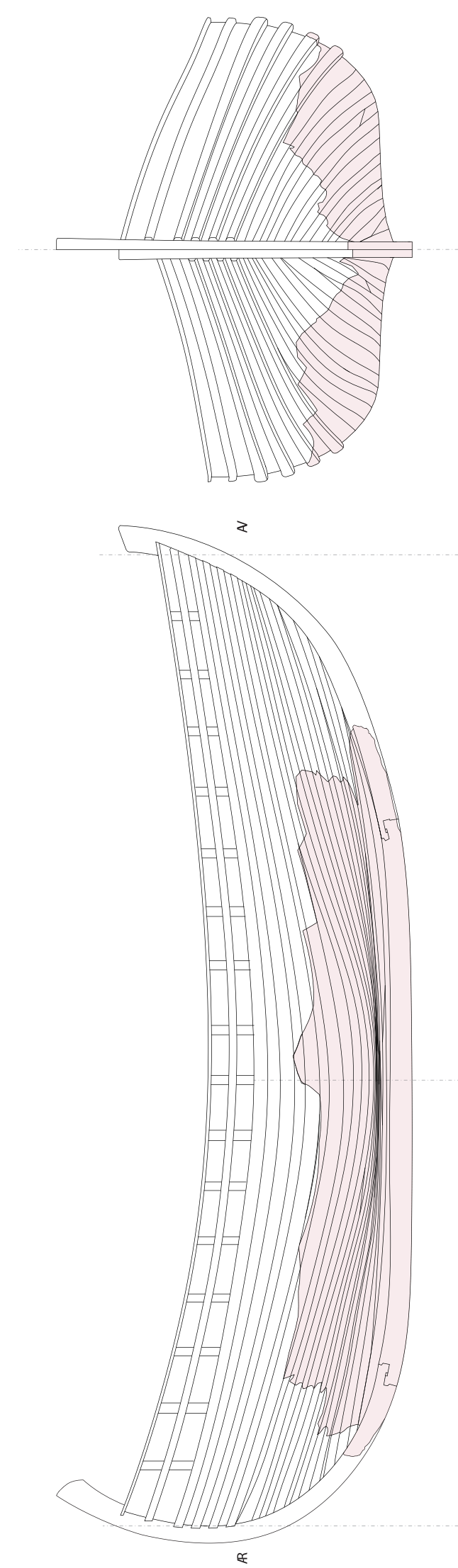
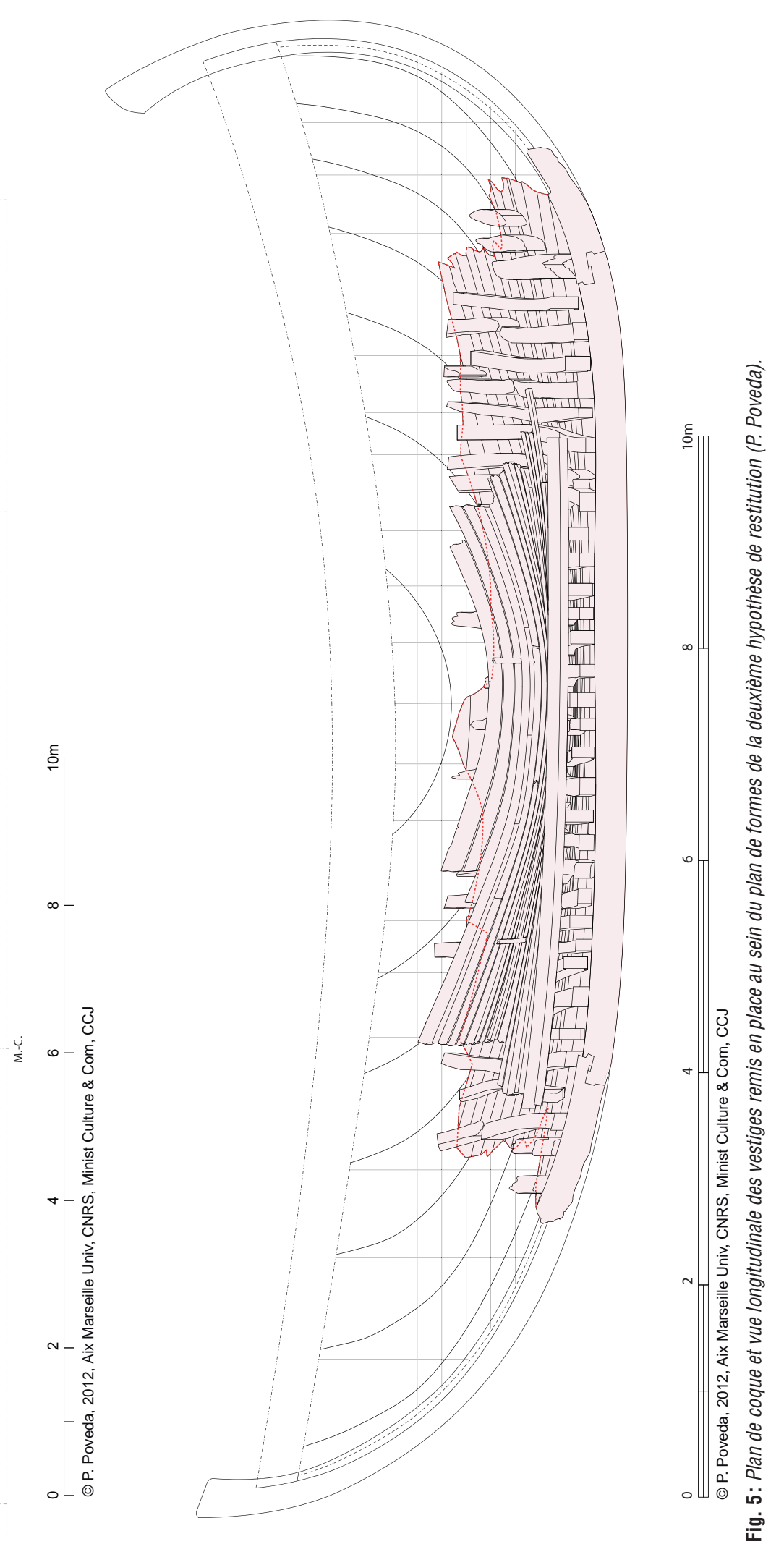


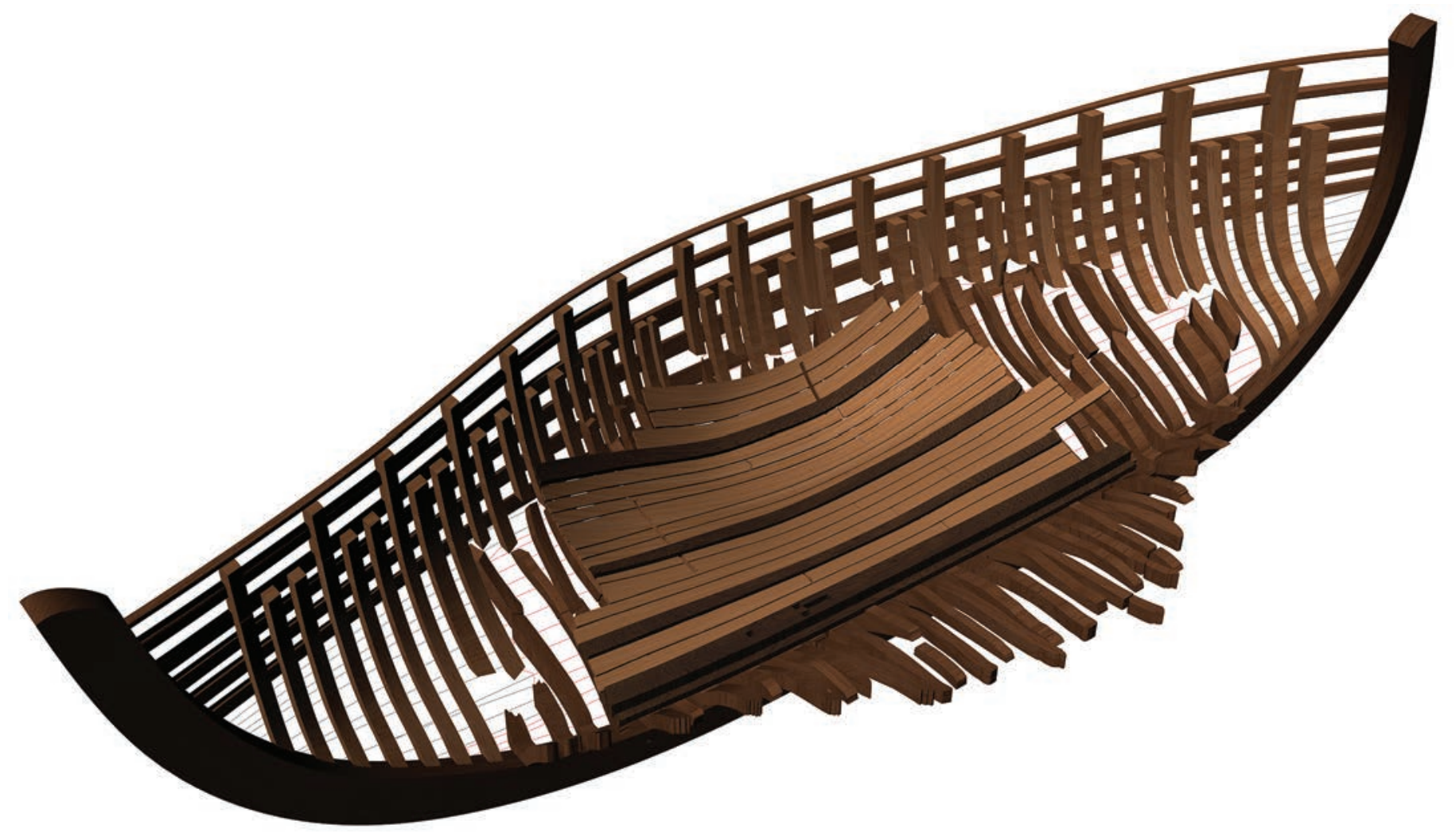

Fig. 6: Vue de trois-quarts du modèle 3D des vestiges et de restitution du flanc tribord (P. Poveda, CCJ/AMU/CNRS).

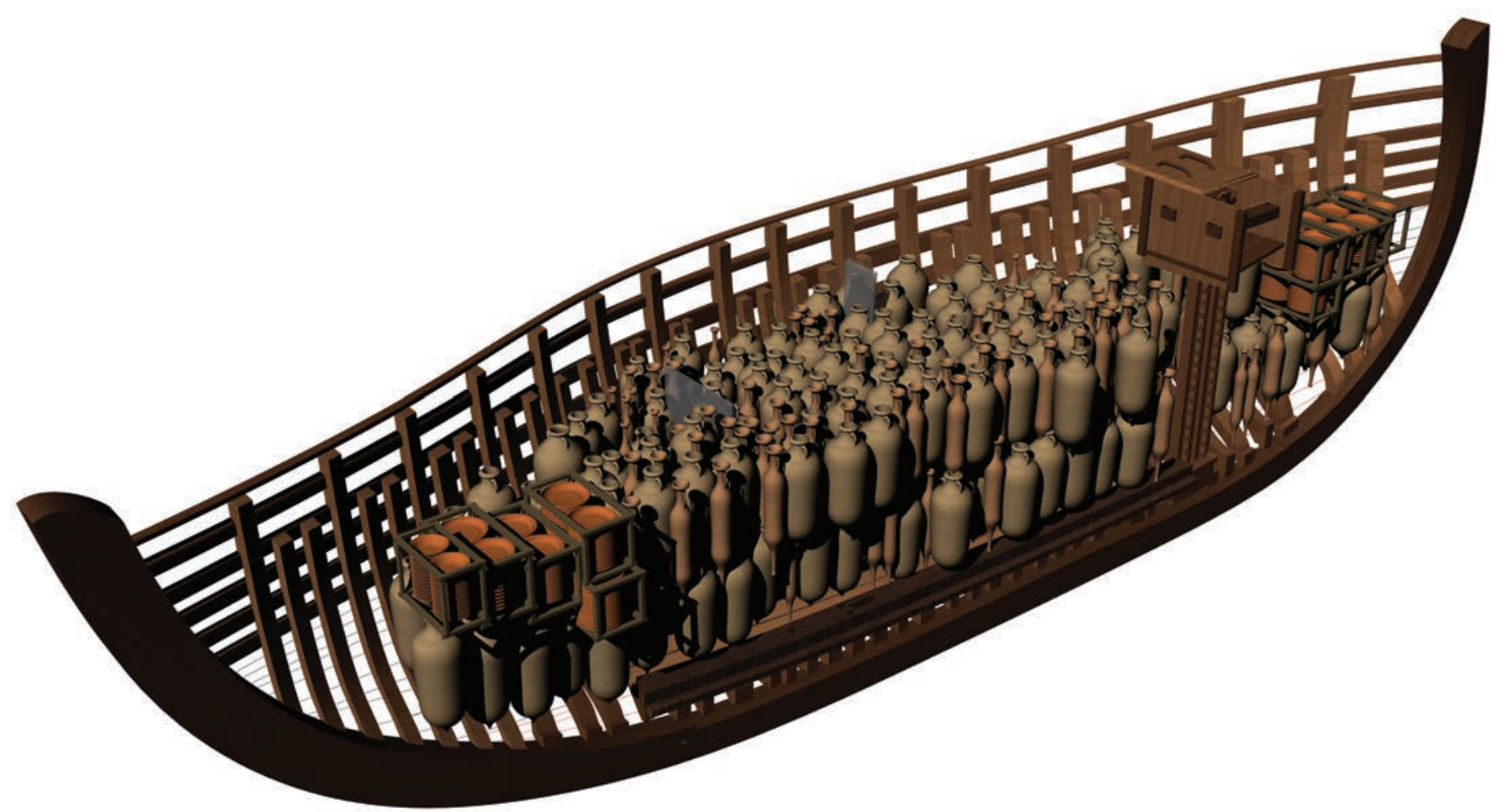

Fig. 7: Vue de trois-quarts du modèle 3D de restitution du flanc tribord et de la cargaison (P. Poveda, CCJ/AMU/CNRS). 
51 amphores Keay XXV ou grands spatheia pour une trentaine conservés. On se rapproche ainsi des estimations graphiques données par Cl. Santamaria (1995, p. 121), le nombre de Keay XXXV étant d'ailleurs identique, même si nos estimations sont plus basses en ce qui concerne les amphores de petit diamètre.

\section{Restitution du deuxième niveau d'amphores}

Dans la deuxième hypothèse de restitution de la carène, l'espace de cale permet de considérer raisonnablement la présence d'un second niveau d'amphores. Contrairement au premier dont le plan a pu être dressé avec précision, nous ne possédons que de très rares informations sur l'importance, la répartition et l'existence même de cette deuxième couche. À partir des quelques photos prises du tumulus lors des premières expertises, il apparaît cependant que la composition de cet éventuel second niveau devait être tout à fait comparable à celle du niveau inférieur: amphores cylindriques de grand diamètre du type Keay XXXV, spatheia et amphores cylindriques de petit diamètre du type Keay XXV. En nous appuyant sur la proportion observée pour le premier niveau entre ces différents types d'amphores, nous avons projeté une couche composée de 106 Keay XXXV et de 87 grands spatheia ou Keay XXV. Une fois encore, nous nous rapprochons de l'estimation graphique évoquée par Cl. Santamaria en ce qui concerne les amphores Keay XXXV (environ 100) et notre estimation s'avère toujours plus basse pour les amphores de diamètre plus réduit (150 selon Santamaria). La manière dont a pu être calée une telle couche demeure énigmatique. Nous avons donc fait le choix, dans la majorité des cas, de placer dans un premier temps les amphores Keay XXXV entre trois ou quatre cols d'amphores du même type du niveau inférieur. De cette manière, nous estimons que les cols des spatheia ainsi que les cols des Keay XXV du premier niveau, intercalés entre des Keay XXXV, devaient tout juste permettre l'insertion des pieds des Keay XXXV du niveau supérieur. Les Keay XXV du niveau supérieur, venant combler l'espace entre les Keay XXXV, pouvaient s'appuyer sur les cols ou sur les épaulements des Keay XXXV du niveau inférieur ou encore venir s'intercaler entre celles-ci.

À ce point, plusieurs remarques importantes peuvent être formulées quant à cette disposition de la cargaison: on note d'une part que les formes creuses de la carène, et donc aussi de la cale, entraînent une variation dans la hauteur d'installation des amphores du premier niveau. Les amphores situées vers les flancs se retrouvant placées bien plus en hauteur que celles du centre, la disposition du second niveau ne peut s'effectuer que dans un volume beaucoup plus réduit, équivalent environ à la moitié de celui du premier niveau. Cette donnée invalide définitivement l'hypothèse d'un second niveau identique au premier comme cela avait pu être avancé, avec beaucoup de prudence toutefois, par Cl. Santamaria.

D'autre part, le remplissage complet de la cale laisse apparaître deux zones de vides qu'il convient de mettre en lumière: il s'agit tout d'abord d'un couloir situé sur les flancs bâbord et tribord, au-dessus des amphores du premier étage, de part et d'autre de la couche formée par les amphores du second niveau. Nous avons fait le choix de laisser cet espace vide étant donné que la place manque en hauteur pour pouvoir y insérer des amphores et qu'aucune autre trace de cargaison ne permet d'envisager le remplissage de cette zone. Si l'on peut considérer que la présence d'espaces vides dans la cargaison pouvait représenter un danger susceptible de remettre en cause la cohésion et l'arrimage du reste du chargement, nous estimons que cette zone de vide est assez réduite pour permettre aux arrimeurs de prévenir un déplacement par quelque moyen comme la liaison des amphores entre elles ou bien encore par le recours à un fardage important. On peut aussi envisager l'aménagement de cloisons comme observé in situ dans la couche inférieure. Un second vide notoire se retrouve aussi au-dessus de la première couche, cette fois-ci vers les extrémités avant et arrière. Dans cette zone, l'espace en hauteur est insuffisant pour placer un deuxième niveau d'amphores et celles qui sont aux alentours définissent un espace vide dont le volume s'apparente à celui d'une pyramide inversée. Comme l'avait évoqué $\mathrm{Cl}$. Santamaria ${ }^{12}$, nous estimons qu'il pourrait s'agir d'un espace dédié à la cargaison des plats de sigillée (Fig. 3 et 7).

\section{Restitution de la cargaison de plats et d'assiettes}

Sur la base des observations menées in situ par Cl. Santamaria et des informations fournies par le modèle, nous avons tenté de replacer dans le modèle tridimensionnel les différentes formes de plats et d'assiettes en sigillée africaine retrouvées lors de la fouille dans les zones vides, mis en évidence aux extrémités du chargement, au-dessus de la première couche.

À partir des formes des plats retrouvés et de la proportion relative de leurs présences, nous avons donc projeté la cargaison suivante: 725 plats parmi lesquels on retrouve environ $60 \%$ de Hayes 61B, $20 \%$ de Hayes 64, $10 \%$ de Hayes 65 et $10 \%$ de Hayes 50B. Compte tenu du pillage important constaté dans cette partie de la cargaison, le degré de précision atteint lors de sa restitution est moindre que celui de la cargaison d'amphores, ce qui doit inciter à considérer avec précaution le nombre total d'individus restitués. De la même manière, il apparaît aussi plus difficile de déterminer avec une bonne précision le positionnement de cette cargaison au sein de la cale: le rangement dans des caisses paraît la solution la plus probable, mais dans ce cas, comme dans celui d'un empilement directement au-dessus des amphores, l'arrimage de l'ensemble demeure incertain.

La disposition finalement proposée dans notre modèle donne à voir une solution dans laquelle l'espacement des caisses et l'empilage des plats remplit complètement, pour la seconde hypothèse de restitution de la carène, l'espace vide laissé entre les amphores du premier étage et le pont (Fig. 3 et 7). Dans le cadre de la première hypothèse, présentant un creux moins important, il apparaît complexe de positionner ces sigillées sur le dessus des amphores du premier niveau, vers les extrémités. De plus, l'espace laissé libre au centre de l'hiloire semblerait, dans un tel cas de figure, une zone mieux adaptée à la réception de cette cargaison secondaire. Dans ce cas, l'arrimage et la répartition des charges au milieu de la cargaison s'avéreraient beaucoup plus logiques.

À ce stade de notre étude, il apparaît difficile de privilégier l'une ou l'autre de ces hypothèses. En effet, la deuxième hypothèse, dans laquelle nous restituons quatre préceintes et un creux de $1,57 \mathrm{~m}$, présente l'avantage de s'accorder parfaitement avec la présence des deux niveaux supposés d'amphores ainsi qu'avec la disposition des céramiques sigillées aux extrémités. Un contre argument est cependant fourni par la présence de nombreux vides en périphérie de la seconde couche d'amphores, vides que l'on

12. «[...] le chargement de céramiques sigillées se trouvait, à l'avant et à l'arrière, sur les amphores du premier niveau, ou à la hauteur de leur partie supérieure. Sans doute en des endroits où l'espace disponible dans la cale ne permettait pas de superposer de nouvelles amphores » (Santamaria 1995, p. 118). 
distingue ainsi particulièrement de part et d'autre des amphores de la deuxième couche sur la section transversale du maître couple sur la figure 3. Or un arrimage consciencieux, que l'on serait en droit d'attendre sur un tel navire, se traduit en effet de préférence par l'absence de vide afin de garantir l'intégrité de la cargaison.

Deux éléments pourraient infirmer l'hypothèse d'un chargement sur un seul niveau: d'une part le manque de hauteur vers les flancs pour insérer les amphores Keay XXXV placées en périphérie; d'autre part le manque d'espace, au-dessus des amphores disposées aux extrémités avant et arrière, pour placer la cargaison de céramiques sigillées dont l'existence semble cependant attestée par les observations effectuées lors de la fouille.

En résumé, il apparaît donc que l'hypothèse de chargement sur deux niveaux, associée à une restitution de la carène avec quatre niveaux de préceintes, rassemble effectivement plus d'arguments que l'hypothèse plus restreinte en hauteur et en chargement (Fig. 7). Toutefois, compte tenu des objections que soulèvent les deux versions, nous avons fait le choix de développer l'analyse hydrostatique et de stabilité pour chacune d'entre elles afin d'affiner notre jugement en vue d'une validation ultérieure.

\section{ANALYSES HYDROSTATIQUES ET DE STABILITÉ}

\section{DEVIS DE POIDS DU NAVIRE EN LÈGE}

L'étude d'identification xylologique conduite sur les bois de l'épave par Frédéric Guibal (CNRS, IMBE UMR 7263) permet, bien qu'elle ne soit pas exhaustive, de définir globalement les divers types d'essences utilisées dans la construction du navire. Ainsi, pour la charpente transversale, si seulement un peu plus de la moitié des éléments ont été effectivement analysés, la répartition des essences reconnues semble présenter un aperçu significatif que l'on peut tenter de reproduire sur les éléments non identifiés. C'est ainsi que nous avons attribué aux éléments sans identification une densité équivalente à celle du noyer, essence largement majoritaire au sein des échantillons prélevés. On notera que la densité des bois formant la carène est ici celle correspondant à une humidité relative du bois de $15 \%$ environ, soit sa densité à l'état sec à l'air. On trouvera ci-dessous un résumé, pour chacune des deux hypothèses, de l'importance et de la répartition des poids pour le navire lège.

\section{DEVIS DE POIDS DE LA CARGAISON}

Nous avons détaillé dans la partie précédente, le nombre d'amphores et de céramiques restituées pour chaque niveau. Pour le devis de poids, il s'agissait donc seulement d'évaluer pour chacun des modules d'amphores le poids et le centre de gravité de chacune d'entre elles.

Comme précisé précédemment, nous avons utilisé quatre modules d'amphores dans le modèle, représentant approximativement les divers modules découverts dans l'épave. La définition de leur poids à vide et de leur contenance provient essentiellement des données fournies et mesurées par $\mathrm{Cl}$. Santamaria directement sur les individus encore conservés et prélevés durant la fouille. Toutefois, le plus grand module de Keay XXXV ainsi que les Keay XXV ont fait l'objet d'une estimation de poids et de volume de notre part. Ainsi, si le module moyen de Keay XXXV peut être évalué à $19 \mathrm{~kg}$ à vide pour une contenance de 60 litres environ, nous estimons que les modules les plus grands peuvent être estimés à $20,9 \mathrm{~kg}$ et contenir 87 litres $^{13}$. Concernant les spatheia et les Keay $\mathrm{XXV}$, leur poids à vide peut être estimé à $10 \mathrm{~kg}$ tandis que leur contenance devait s'établir aux environs de 10,5 l en moyenne. Les petits spatheia présentent, quant à eux, un poids à vide de $5 \mathrm{~kg}$ pour une contenance de 51 environ.

Pour le devis de poids, il fallait aussi intéresser au contenu des amphores ainsi qu'à la densité de ces contenus. Cependant, il est très vite apparu que, malgré l'hétérogénéité des marchandises transportées, on constate une certaine homogénéité des densités. En effet, mis à part la densité d'un contenu d'olives, qu'il nous a été impossible de mesurer, nous avons constaté que les autres contenus potentiels (salaisons de viande ou de poisson, huile, vin) présentaient tous des densités légèrement supérieures ou inférieures à 1 , permettant d'arrondir à cette densité celle de l'ensemble de la cargaison. Par défaut, nous avons donc maintenu une densité de $1 \mathrm{~kg}$ / $\mathrm{dm}^{3}$. En tenant compte de la répartition finale du chargement proposée dans notre modèle de restitution, le poids du premier niveau d'amphores peut être estimé à 21,25 tonnes, tandis que le second niveau peut être estimé à 10,15 tonnes. La cargaison de sigillées peut, quant à elle, être évaluée à environ 1 tonne.

Au final, les deux hypothèses de chargement associées aux deux hypothèses de restitution de la carène donnent donc à voir deux navires sensiblement différents au regard de leurs capacités nautiques et de leurs capacités commerciales. Dans le premier cas, le navire présente un déplacement total de 31,30 tonnes contre 42,16 tonnes pour le second. Toutefois, on remarque que le rapport entre le port en lourd et le déplacement lourd varie finalement assez peu entre ces deux hypothèses, de $71,1 \%$ à $76,9 \%$. De la même manière, les enfoncements respectifs dans l'eau ne semblent pas révéler des situations fort différentes: dans un cas comme dans l'autre le franc-bord en charge correspond grossièrement à la distance entre le livet et l'avant dernière préceinte (Fig. 8). L'analyse hydrostatique de chacune de ces conditions et de la stabilité transversale de celles-ci nous permet cependant de distinguer entre elles des différences non négligeables en ce qui concerne la navigation et les capacités de navigation.

\section{ANALYSE DE LA STABILITÉ ET DE LA RAIDEUR À LA TOILE}

Le principe de base de nos analyses sur cette stabilité repose sur une simple comparaison entre le moment de redressement transversal, généré à la gîte par la forme de la carène et par la répartition des poids ${ }^{14}$, et le couple inclinant engendré par la pression du vent dans la voilure. En d'autres termes, il s'agit de déterminer la raideur à la toile du navire et d'évaluer sa capacité d'évolution dans diverses conditions de vent. Notons que cette stabilité dynamique étant le résultat d'une multitude de forces appliquées au navire, celle-ci est difficile à cerner avec exactitude. De ce fait, le résultat de nos

13. Le poids des conteneurs céramiques et leur contenance ont été établis grâce à la modélisation 3D. Cette étape permet de définir très précisément tout à la fois le volume interne des amphores tout comme la quantité d'argile les constituant. L'application, à ces volumes, des coefficients correspondant à la masse volumique des produits contenus et au corps céramique du conteneur permet de définir, finalement, le poids total de l'amphore pleine.

14. La stabilité transversale d'un navire se décompose généralement entre la stabilité de poids (situation en hauteur du centre de gravité) et la stabilité de formes (engendrée par la largeur de la carène et par le déplacement latéral à la gîte du centre de carène). 

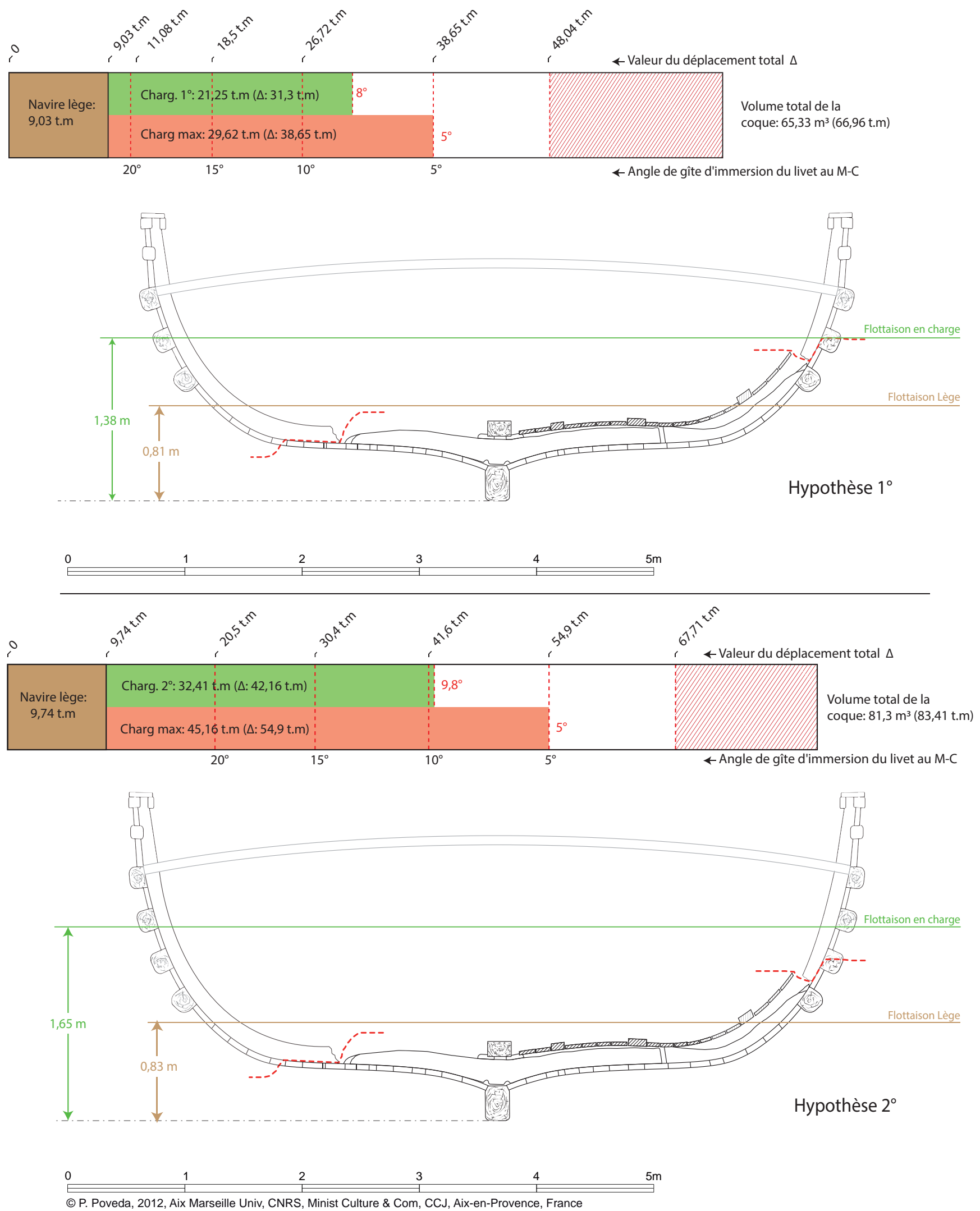

Fig. 8: Diagrammes représentant les différents déplacements des deux hypothèses et représentations graphique des lignes de flottaison des deux hypothèses au niveau du maître-couple (P. Poveda). 
analyses n'est qu'une estimation dont l'approximation générale nous semble satisfaisante pour notre type d'étude.

Dans le cas de Dramont $E$ nous avons testé pas moins de six configurations de chargement sur chacune des deux hypothèses. Nous ne reviendrons cependant ici que sur les résultats des analyses des deux hypothèses de restitution principales en configuration de charge (Fig. 8).

\section{Hypothèse 1: trois niveaux de préceinte et une unique couche d'amphores}

Dans cette configuration, le navire présente un enfoncement important (de 1,38 m contre seulement $0,81 \mathrm{~m}$ en lège, le franc-bord s'établissant alors à $0,44 \mathrm{~m}$ contre $1,02 \mathrm{~m}$ en lège). On constate alors un moment de redressement important (max. $0,57 \mathrm{~cm}$ à $24,2^{\circ}$ de gîte) lui permettant d'étaler un vent de 30 nœuds environ. Toutefois, contrairement à la navigation en lège, l'accroissement important de l'enfoncement réduit de façon inquiétante la réserve de flottabilité au point qu'une gîte de $8^{\circ}$ seulement suffit à l'immersion du livet, l'envahissement intervenant quant à lui vers $24^{\circ}$. Si une telle configuration ne peut pas être jugée comme tout à fait critique, on peut néanmoins considérer que la marge de sécurité raisonnable est atteinte, voir déjà dépassée.

Cette première hypothèse présente donc un navire, qui malgré des rapports de proportions extrêmes (allongement de 2,17 ; rapport largeur/creux de 4,36), peut être considérée comme vraisemblable. Cependant, le port-en-lourd proposé ici, mesuré sur la base d'une cargaison constituée a minima par les éléments ayant échappé au pillage, révèle une situation de chargement relativement critique si l'on doit effectivement considérer un parcours de Dramont $E$ depuis les côtes africaines jusqu'aux rivages de la Narbonnaise. Les marges de sécurité, comme le franc-bord de $44 \mathrm{~cm}$, sont extrêmement réduites pour un navire amené à naviguer en haute mer sans pouvoir anticiper les conditions rencontrées.

\section{Hypothèse 2: quatre niveaux de préceintes et deux couches d'amphores}

Dans cette seconde configuration, la cale est désormais pleine et le port-en-lourd s'élève à 32,4 tonnes. Le déplacement du navire qui s'élève à 42,16 tonnes entraîne un enfoncement important qui porte le tirant d'eau du navire à 1,65 m, le franc bord n'étant plus que de $0,54 \mathrm{~m}$. En ce qui concerne la stabilité transversale, si le bras de levier est moins grand que dans la configuration précédente (max. $0,51 \mathrm{~m}$ atteint à $28,5^{\circ}$ de gîte), le poids total du navire engendre un moment de redressement plus important qui permet au navire d'étaler des vents, jusqu'à 35 nœuds. La hauteur de franc-bord conservée garantit par ailleurs une réserve de flottabilité plus importante: le livet ne se trouvant immergé que vers $10^{\circ}$ de gîte et l'envahissement n'intervenant qu'à $28,5^{\circ}$.

Contrairement donc au chargement sur un seul niveau de la première hypothèse, on peut considérer que cette configuration s'avère plus raisonnable du point de vue de la sécurité à la mer. La situation de charge demeure cependant importante et comporte une part de risques significative pour une traversée de la Méditerranée.

L'analyse de stabilité permet donc d'avancer un ultime argument en faveur de cette seconde hypothèse de restitution et de chargement. Il s'agit indéniablement d'une configuration plus sûre que celle proposée premièrement et ce, bien que l'on demeure dans des limites de sécurité très ténues induites par un chargement extrêmement important.

\section{CONCLUSION}

Les données qui ressortent de l'analyse hydrostatique et de stabilité des restitutions proposées semblent désigner la seconde hypothèse comme la plus vraisemblable. Celle-ci rassemble de fait le plus d'arguments en sa faveur, tant en ce qui concerne la cohérence structurelle du navire que la restitution de la cargaison et de l'analyse hydrostatique et de stabilité.

Du point de vue de la méthode et des outils mis en œuvre, on retiendra que c'est le recours à l'imagerie virtuelle, et aux outils informatiques plus généralement, qui a permis de dresser un tel constat.

Concernant l'interprétation de ces résultats du point de vue archéologique, il nous semble que de telles restitutions portent un éclairage supplémentaire et différent sur les pratiques commerciales maritimes. Compte tenu de la nature très homogène de la cargaison, il y avait en effet tout lieu de penser que le navire devait arriver directement depuis la région de Nabeul ${ }^{15}$. L'ensemble des analyses hydrostatiques et de stabilités semblent d'ailleurs le confirmer: il apparaît tout à fait possible, étant donné les conditions de chargement, d'enfoncement et de stabilité, d'envisager une traversée Sud-Nord de la Méditerranée depuis la province d'Afrique jusque vers les rivages de la Narbonnaise. Mais, à cette première constatation viennent aussi s'ajouter des pistes de réflexions nouvelles. Ainsi, si l'on ne peut, à partir de l'ensemble des données rassemblées, définir la cause précise du naufrage, on peut néanmoins relever quelques éléments remarquables qui ont pu avoir une influence sur le sort du navire: sa grande surface mouillée et sa forme particulièrement renflée devaient lui procurer une inertie certaine le rendant difficilement manœuvrable dans le petit temps. Sa grande largeur devait lui procurer une importante raideur à la toile apte à fatiguer inhabituellement sa structure. A cela, vient s'ajoute enfin une surcharge certaine qui touche aux limites du raisonnable en termes de sécurité pour un navire destiné à de grandes navigations.

Pierre PovedA Ingénieur de recherche Aix Marseille Univ, CNRS, Minist Culture, CCJ, poveda@mmsh.univ-aix.fr

15. Sans revenir sur le détail de la cargaison, notons que selon Michel Bonifay (CCJ-CNRS, UMR 7299) l'essentiel des amphores provient d'ateliers de la région de Nabeul, tout comme la plupart des céramiques sigillées du chargement complémentaire (Bonifay 2004, p. 453). 


\section{BIBLIOGRAPHIE}

\section{BONIFAY M.}

2004 Études sur la céramique romaine tardive d'Afrique, Oxford, Archaeopress (BAR International Series, 1301).

\section{Chevalier Y., Santamaria Cl.}

1970 Note technique sur la couverture photographique réalisée sur l'épave antique du Bas-Empire au cap Dramont, Forma Maris Antiqui, VII, p. 1-8.

JONCHERAY J.-P.

1975 L'épave E du cap Dramont: sigillées claire D et amphores rescapées du pillage, Cahiers d'archéologie subaquatique, IV, p. 141-146.

Gassend J. M., Liou B., Ximenes S.

1984 L'épave 2 de l'anse des Laurons (Martigues, Bouches-du-Rhône), Archaeonautica, 4, p. 76-105.

Pomey P.

1998 Conception et réalisation des navires dans l'Antiquité méditerranéenne, dans E. Rieth (dir.), Concevoir et construire les navires. De la trière au picoteux, Technologie, Idéologies, Pratique, Revue d'anthropologie des connaissances, XIII-1, p. 49-72.

2004 La structure du navire de la Madrague de Giens et le type hellénistique, Ligures, Rivista di Archeologia, Storia, Arte e Cultura Ligure, 2, p. 370-373.

Pomey P., Rieth E.

2005 L'archéologie navale, Paris, Errance.

Pomey P., Kahanov Y., Rieth E.

2012 Transition from Shell to Skeleton in Ancient Mediterranean ShipConstruction: analysis, problems, and future research, The Inter- national Journal of Nautical Archaeology, 41.2, septembre 2012, p. 235-314.

Poveda P.

2012a Le navire antique comme instrument du commerce maritime: restitutions 3D, tonnage, qualités nautiques et calculs hydrostatiques des épaves: Napoli A, Napoli C, Dramont E et Jules-Verne 7, Thèse de doctorat sous la direction de P. Pomey, Aix-Marseille Université, Aix-en-Provence, non publiée.

2012b Hypothetical reconstruction of the Dramont E Shipwreck, dans Günsenin N. (dir.), Between continents: proceedings of the XIIth International symposium on boat and ship archaeology, Istanbul, 2009, Istanbul, Ege Yayinlari 5 (ISBSA, 12), p. 331-336.

\section{SANTAMARia Cl.}

1983 Note sur la partie inférieure du mât du navire antique du Dramont E, Annales du Sud-Est Varois, VIII, p. 57.

1984 Le pied de mât de l'épave «E» du cap Dramont (Saint-Raphaël, Var), Archaeonautica, 4, p. 107-114.

1995 L'épave Dramont «E» à Saint-Raphaël (ve siècle ap. J.-C.), Paris, éditions du CNRS (Archaeonautica 13).

\section{TChernia A.}

1969 Informations archéologiques: recherches sous-marines, Gallia, XXVII, p. 465-499.

Tchernia A., Pomey P., Hesnard A.

1978 L'épave romaine de la Madrague de Giens (Var), Paris, éd. du CNRS (34e Suppl. à Gallia, 34). 